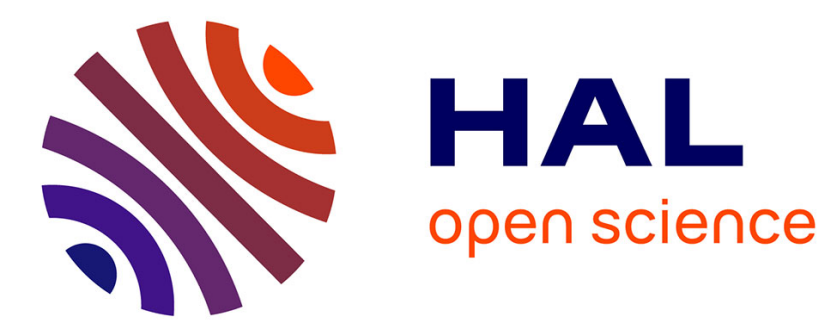

\title{
Attosecond control of collective electron motion in plasmas
}

Fabien Quéré

\section{To cite this version:}

Fabien Quéré. Attosecond control of collective electron motion in plasmas. Nature Physics, 2012, doi:10.1038/nphys2269. 10.1038/nphys2269 . hal-00716202

\section{HAL Id: hal-00716202 \\ https://hal.science/hal-00716202}

Submitted on 10 Jan 2013

HAL is a multi-disciplinary open access archive for the deposit and dissemination of scientific research documents, whether they are published or not. The documents may come from teaching and research institutions in France or abroad, or from public or private research centers.
L'archive ouverte pluridisciplinaire HAL, est destinée au dépôt et à la diffusion de documents scientifiques de niveau recherche, publiés ou non, émanant des établissements d'enseignement et de recherche français ou étrangers, des laboratoires publics ou privés. 


\title{
Attosecond control of collective electron motion in plasmas
}

\author{
Antonin Borot ${ }^{1, *}$, Arnaud Malvache ${ }^{1, * \dagger}$, Xiowei Chen $^{1}$, \\ Aurélie Jullien ${ }^{1}$, Jean-Paul Geindre ${ }^{2}$, Patrick Audebert ${ }^{2}$, \\ Gérard Mourou ${ }^{3}$, Fabien Quéré ${ }^{4}$, Rodrigo Lopez-Martens ${ }^{1}$
}

\footnotetext{
${ }^{1}$ Laboratoire d'Optique Appliquée, ENSTA-Paristech, Ecole Polytechnique, CNRS, 91761 Palaiseau, France

${ }^{2}$ Laboratoire pour l'Utilisation des Lasers Intenses, Ecole Polytechnique, CNRS, 91128 Palaiseau, France

${ }^{3}$ Institut de la Lumière Extrême, ENSTA-Paristech, Ecole Polytechnique, CNRS, 91761 Palaiseau, France

${ }^{4}$ Service des Photons, Atomes et Molécules, CEA, DSM/IRAMIS, CEN Saclay, 91191 Gif-sur-Yvette, France

${ }^{\dagger}$ Correspondence should be addressed to: A.M.
}

Today, light fields of controlled and measured waveform can be used to guide electron motion in atoms and molecules with attosecond precision. Here, we demonstrate attosecond control of collective electron motion in plasmas driven by extreme intensity $\left(\approx 10^{18} \mathrm{~W} / \mathrm{cm}^{2}\right)$ light fields. Controlled few-cycle near-infrared light waves are tightly focused at the interface between vacuum and a solid-density plasma, where they launch and guide subcycle motion of electrons from the plasma with characteristic energies in the multi-kiloelectronvolt range - two orders of magnitude more than what has been achieved so far in atoms and molecules. Basic spectroscopy of the coherent extreme ultraviolet radiation emerging from the light-plasma interaction allows us to probe this collective motion of charge with sub-100-attosecond resolution. This is an important step towards attosecond control of charge dynamics in laser-driven plasma experiments.

Two major trends can nowadays be identified in the interaction of ultrashort laser pulses with matter. On the one hand, ultrahigh light intensities provided by multi-terawatt femtosecond lasers can be used to drive collective electron motion in plasmas up to the 0.1-1 gigaelectronvolt energy range [1], opening the way to very compact laser-based particle accelerators for nuclear and medical applications [2]. On the other hand, controlled few-cycle light waves can be used at moderate intensities to drive and probe the attosecond dynamics of few-electron motion in atoms $[3,4,5,6]$, molecules $[7,8]$ and condensed matter $[9,10]$ - with typical energies

* These authors contributed equally to this work. 
ranging between tens to a few hundred electronvolts [11].

Merging these two trends, i.e. using tailored waveforms of extreme intensity light to steer the collective motion of high-energy plasma electrons, will open brand new perspectives for imaging ultrafast charge dynamics during extreme intensity laser-plasma interactions. First experiments have already highlighted the need for waveform control when trying to reproducibly guide attosecond electronic processes in plasmas with intense few-cycle light fields [12]. For the first time, we use fully controlled few-cycle near-infrared (NIR) light fields of extreme intensity $\left(10^{18} \mathrm{~W} / \mathrm{cm}^{2}\right)$ to reproducibly launch and probe collective electron motion at the interface between vacuum and a solid-density plasma with attosecond precision (Fig. 1a-b).

\section{Light-driven plasma mirrors}

When an intense femtosecond laser pulse interacts with a solid, its rising edge strongly ionizes the surface atoms, creating a layer of plasma with near-solid electronic density $\left(\sim 10^{23} \mathrm{~cm}^{-3}\right)$, which becomes highly reflective a so-called plasma mirror - for light at wavelengths greater than a few tens of nanometers $[13,14,15,16,17]$. During the interaction with the pulse, the plasma layer can only expand by a small fraction of the optical laser wavelength, $\lambda_{L}$, which leads to the formation of a very sharp interface with vacuum extending over a distance $\ll \lambda_{L}$ (Fig. 1b), typically of the order of a few tens of nanometers. At this steep plasma-vacuum interface, the laser field behaves like in the usual case of light reflection at a metallic surface. The total electric-field inside the plasma is zero due to screening by the dense conducting plasma.

On a macroscopic scale, the boundary conditions on the oscillating electromagnetic field at the interface imply that the component $E_{t}$ of the laser electric field parallel to the surface is continuous and therefore vanishes. In contrast, its component $E_{n}$ normal to the surface can exhibit a discontinuity : it has an oscillation amplitude that jumps from 0 , in the conducting medium, to a maximum of $2 E_{L} \sin \theta$, where $\theta$ is the incidence angle of the laser with respect to the target normal. At light intensities close to $10^{18} \mathrm{~W} / \mathrm{cm}^{2}$, the amplitude of the laser electric-field normal to the plasma-vacuum interface can thus exceed the teravolt per meter and therefore accelerate electrons from the plasma up to very high kinetic energies on the time scale of a single optical cycle.

On a microscopic scale, there is of course no such field discontinuity. Electrons at the plasma surface are periodically dragged in and out of the target by the $E_{n}$ component of the oscillating laser field, while the much heavier ions of the plasma are left behind. This creates a charge separation field on the time scale of the laser cycle, which screens $E_{n}$ over a finite length. For everyday light intensities, this screening length is very small, typically on the Angström scale, hence justifying the usual boundary condition at conductor surfaces. In the intense laser field, however, the excursion of surface electrons into vacuum can reach several tens of nanometers

(Fig. 1b) - a significant fraction of the laser wavelength - with kinetic energies of the order of the ponderomotive energy $U_{p}=e^{2} E_{n}^{2} / 4 m \omega_{L}^{2}$ ( $e$ and $m$ electron charge and mass, $\omega_{L}$ laser angular frequency) in the light 
field - typically several tens of kiloelectronvolts $(\mathrm{keV})$ for light intensities in the range $10^{17}$ to $10^{18} \mathrm{~W} / \mathrm{cm}^{2}$. In our experiment, we probe this large-amplitude collective motion of charge with sub-100-as resolution. This we achieve by recording the laser waveform-dependent emission of extreme-ultraviolet (XUV) light triggered by the subcycle motion of the surface electrons within the solid plasma layer.

\section{Coherent wake emission}

As shown in Fig. 1b, electrons at the plasma surface are pulled out into vacuum when, during each laser cycle, the force $-e E_{n}$ exerted by the laser electric-field points toward vacuum. Later in the laser cycle, as $E_{n}$ decreases in magnitude, some of the electrons involved in the screening are pushed back towards the dense plasma because the space charge field they experience from other electrons exceeds the laser field $E_{n}$. Finally, when $E_{n}$ changes sign, all the electrons are accelerated back towards the plasma layer. Once they pass the region of the plasma above critical density $N_{c}$, where the laser light is screened by the high electronic density, they escape the grip of the laser field and travel field free. For $800 \mathrm{~nm}$ light, this occurs at a depth $x$ in the plasma, where the local density is $n(x)>N_{c}=m \epsilon_{0} \omega_{L}^{2} / e^{2}=1.7 \times 10^{21} \mathrm{~cm}^{-3}$ (Fig. 1b). During this process, electrons carry away the energy they acquired in the light field: this is the so-called Brunel absorption $[18,19]$. The combined effect of both the laser and space charge fields is to make faster electrons return to the plasma surface at later times during the laser cycle. In the overdense region of the plasma, however, faster electrons can now freely catch up with slower moving electrons that returned earlier during the cycle: as a result, Brunel electrons pulled out of the plasma around the same time in the laser cycle eventually bunch together to form a local electron density peak of a few $N_{c}$ inside the plasma [20]. Lower energy electrons bunch earlier at lower electronic densities closer to the plasma surface and higher energy electrons bunch later at higher electronic densities deeper inside the plasma layer. This electron density peak has a typical duration of the order of $100 \mathrm{as}$ and travels across the plasma at typical velocities ranging from 0.1 to 0.3 times the speed of light for the laser intensities considered here [20]. In its wake, it pushes the background plasma electrons that collectively oscillate at the local plasma frequency $\omega_{p}(x)=\sqrt{n(x) e^{2} / m \epsilon_{0}}$. In the steep plasma density gradient, these plasma oscillations, which are initially longitudinal electrostatic modes, can couple to transverse electromagnetic modes and thus radiate light at the plasma frequency $\omega_{p}(x)[21]$. This can be seen as the time-reverse of resonant absorption [22], where laser light is partially converted into collective electron oscillations in a gradient of plasma density (both effects correspond to linear mode conversion mechanisms $[22,23])$. This emission, known as coherent wake emission or CWE [24], consists of a sub-femtosecond burst of coherent radiation, superimposed with the laser light reflected at the plasma surface [25], with a spectrum extending into the extreme ultraviolet $(\mathrm{XUV})$ up to $\omega_{\max }=\sqrt{N_{\text {solid }} / N_{c}} \omega_{L}$, emitted from the region of solid plasma density $N_{\text {solid }}$ (Fig. 1b). Experimentally, the measured CWE spectrum results from the interference 
in time of successive light bursts triggered by the attosecond bunching of returning Brunel electrons inside the plasma with each laser cycle (Fig. 1c-d). The total emission spectrum can therefore be expressed as :

$$
S(\omega)=\left|\sum_{j=1}^{N} s_{j}(\omega) e^{i \omega \tau_{j}}\right|^{2}
$$

where $s_{j}(\omega)$ is the complex spectrum emitted in the $j^{t h}$ laser cycle, corresponding to an attosecond pulse centered at time $\tau_{j}$. When driven by a laser pulse containing many wave cycles, CWE consists of a long train of attosecond pulses - one per laser cycle - and a measured XUV spectrum made up of odd and even harmonics of the laser frequency $\omega_{L}$. In our experiment, we measure the CWE spectrum generated by a laser waveform containing only a few controlled oscillations of NIR light. We exploit the fact that the waveform-driven collective dynamics of Brunel electrons are directly mapped onto the time structure of the emitted attosecond pulse train or APT (Fig. 1b-c) and that even subtle changes in the subcycle charge dynamics induced by different NIR waveforms can lead to dramatic changes in the measured XUV spectrum (Fig. 1d)[12].

\section{Waveform-dependent emission spectra}

In the experiment, we use p-polarized 5-femtosecond (FWHM), 0.8-micron wavelength laser pulses focused at oblique incidence down to $1.7 \mu \mathrm{m}$ spot size onto a moving optically polished glass surface [26] at peak light intensities close to $10^{18} \mathrm{~W} / \mathrm{cm}^{2}$ (more details in supporting online materials). The waveform of the laser electric-field can be written in complex form as $E_{L}(t)=E_{0} a_{L}(t, \alpha) e^{-i\left(\left(\omega_{L}+\alpha t / 2 \tau_{0}^{2}\right) t+\phi_{0}\right)}$, where $E_{0}$ is the peak electric-field strength, $a_{L}(t, \alpha)$ is the normalized amplitude envelope, $\alpha$ the linear frequency chirp of the pulse, $\tau_{0}$ the Fourier-transform limited pulse duration, $\phi_{0}$ its carrier-envelope (CE) phase (defined on Fig. 1c) and $\omega_{L}$ the carrier wave frequency (the variation of the pulse temporal width $\tau$ with $\alpha$ is detailed in supporting online materials). The CE phase drift of the laser system can be stabilized down to 200-milliradian (rms), corresponding to a sub-100-as jitter of the few-cycle NIR light waveform with respect to the pulse envelope.

Fig. 2b shows the measured CWE spectra in the $7 \omega_{L}-10 \omega_{L}$ spectral range as a function of the relative CE phase of Fourier-transform limited 5-fs laser pulses, i.e. with zero frequency chirp. Peaks separated by $\omega_{L}$ are clearly observed, as one would expect in experiments using a many-cycle laser waveform. However, as the $\mathrm{CE}$ phase of the pulse changes, the positions of these harmonic-like peaks no longer correspond to integer multiples $n \omega_{L}$ of the laser frequency, but instead linearly drift with a slope of $\approx \omega_{L} / 2 \pi$. Fig. 2 a-c-d display the same measurements performed for different frequency chirps, both positive and negative. These results show that the effect of the CE phase on the CWE spectrum also depends on the frequency chirp of the NIR waveform. For $\alpha<0$, the peaks are slightly narrower and their position still drifts linearly with relative CE phase, while just changing the sign of the chirp $(\alpha>0)$ almost suppresses the effect of the CE phase on the measured position of the peaks in the spectrum. The reproducible changes in the plasma emission spectrum 
induced by only $\pi / 10$ changes in the $\mathrm{CE}$ phase of the driving laser field show that we control the collective plasma electron dynamics underlying CWE with sub-200 attosecond precision.

\section{Collective attosecond electron dynamics}

The spectroscopy of CWE provides information on the collective attosecond dynamics of Brunel electrons. To demonstrate this, we developed a simple model, inspired from the one developed in [18] and similar to the one described in [12], which gives direct insight into the measurements of Fig. 2. This model consists in solving the relativistic equation of motion for a collection of electrons, assuming one-dimensional motion along the target normal. In this equation, the only forces taken into account, when the electrons are in vacuum, are the one exerted by the laser electric-field $E_{n}$ normal to the target and by the space charge field induced by the collective plasma response to the laser. Once in the plasma, electrons are considered to experience no force at all. From the obtained set of electron trajectories, illustrated in Fig. 1b, we determine the crossing times of electron trajectories inside the plasma layer for each laser cycle (more details in supporting online materials). This collection of subcycle crossing times in turn dictates the relative timing of pulses in the APT emitted by the background plasma oscillations. Because in CWE, the shape of the attosecond pulse is almost independent of the amplitude of the light wave, the time structure of the APT is almost exclusively determined by the relative timing of XUV emission from one wave cycle to the next (Fig. 1c), and this model allows us to reconstruct the temporal structure of CWE with attosecond resolution.

Fig. 3d to $\mathrm{f}$ show the temporal intensity profile of the APTs predicted by our model as a function of the CE phase $\phi_{0}$ of the three different NIR waveforms (Fig. 3a to c). The intensity of the APTs are plotted as a function of $t / T_{L}+\phi_{0} / 2 \pi$ (horizontal axis): a vertical line in this plot therefore corresponds to an event that drifts linearly in time with changing CE phase. Fig. 3 shows that the main effect of the CE phase is to shift the temporal position of APT relative to the pulse envelope. This is because the subcycle motion of the electrons is temporally locked to the NIR waveform, and a change of $\Delta \phi$ in the CE phase thus delays the electron trajectory crossing times inside the plasma by $\approx \Delta \phi / \omega_{L}$, which is directly mapped onto the APT structure. More generally, by controlling the waveform of the NIR driving light, we can control the electron crossing time, and thereby the timing of emission of XUV light by the plasma, with sub-100-as precision.

\section{Moiré patterns}

Measuring the sensitivity of CWE spectra (Fig.2) to the waveform of the driving light provides a measure of how accurately we can control the ultrafast charge dynamics unfolding within the plasma. The sensitivity of the harmonic generation process to the CE phase results from the combination of two effects. First, due to the global temporal drift of the APT it induces, a change in the CE phase changes the intensities at which each individual attosecond XUV burst in the APT is generated. Second, a change in intensity modifies the subcycle 
emission time of each XUV burst because it changes the time it takes for the returning Brunel electrons to reach the dense part of the plasma where they cross and trigger CWE $[27,28]$. For a few-cycle pulse envelope, the combination of these two effects leads to a dependence of the APT time structure on the CE phase of the pulse. This effect is clearly visible in Fig. 3d-e, where the temporal spacings between individual attosecond pulses (i.e. the distances between vertical stripes) vary with the CE phase of the driving waveform. Using our model, we can calculate the whole CWE spectrum (from $2 \omega_{L}$ to $\omega_{p}^{\max }$ ), i.e. the Fourier transform of the APT of Fig. 3e , as a function of CE phase. The outcome of this calculation, displayed in Fig. 4a, is compared to the results of a much more comprehensive simulation, carried out using a 2D particle-in-cell (PIC) code (more details in supporting online materials), in Fig. 4b, showing a remarkable agreement. In both cases, spectra exhibit a central portion consisting of well-resolved harmonic-like peaks whose position in frequency linearly drifts with CE phase. This central portion corresponds to our experimental spectral detection window and here our model perfectly matches the experiment (Fig. 2b).

Another feature revealed by our model is that outside the experimental detection window, at both ends of the spectrum, the harmonic-like structure of CWE becomes blurred but still exhibits the same periodic dependence on the CE phase of the laser pulse. This global structure corresponds to a moiré pattern, a wellknow interference effect ubiquitously observed in nature [29]. Indeed, a pair of attosecond pulses spaced by $\Delta t_{i}=\tau_{i+1}-\tau_{i}=T_{L}+\delta t_{i}$ (with $T_{L}$ the laser period) in the APT produces a sinusoidal interference pattern in the frequency domain of periodicity $\Delta \omega_{i}=2 \pi / \Delta t_{i}$. The total spectrum originates from the superposition of such interference patterns between all the different pairs of attosecond pulses generated during the light-plasma interaction. It is only when these sinusoidal patterns are in phase that well-contrasted harmonic-like peaks occur in the spectrum. In our case, considering the simple spectral interference pattern created by the beating of the three most intense XUV light bursts in the APT for different values of CE phase (Fig. 4c-d) is enough to qualitatively understand the complex features of the whole CWE spectra of Fig. 4, as well as the drift of the harmonic-like peaks with the CE phase, observed experimentally for a Fourier-transform limited laser waveform (5-fs laser pulse). This phenomenon also explains why the well-contrasted peaks in the spectrum cannot be strictly interpreted as harmonic of the driving laser frequency.

In the harmonic spectra, the positions of the harmonic-like peaks corresponding to contrast revival in the moiré pattern, which depend on the temporal spacings between different pairs of attosecond pulses in the APT are therefore directly determined by the waveform of the laser light. This is how our experiment probes the temporal drift of the APT as a function of CE phase. Experiments carried out for different frequency chirps support this interpretation. For a non-chirped laser waveform, the time spacing between neighboring attosecond pulses naturally increases in time during the laser pulse, regardless of the CE phase value (Fig. 3e). This effect can be compensated by using a positively chirped laser waveform, for which the linearly decreasing light period from one wave cycle to the next balances the increasing delay between successively generated attosecond 
pulses induced by the temporal variation of the pulse envelope. Under these conditions, an almost perfectly periodic train of evenly spaced attosecond pulses can be generated (Fig. 3f). Here, as the CE phase changes, the position of the whole APT still drifts with respect to the pulse envelope as before but this drift no longer leads to changes in its temporal structure. This is why for a positively chirped waveform, the experimental CWE spectrum exhibits very little dependence on the CE phase (Fig. $2 \mathrm{~g}$ and $\mathrm{h}$ ). In contrast, a negative frequency chirp in time throughout the pulse will increase the uneven time spacing between pulses in the APT (Fig. 3d). The dependence of the CWE spectrum on the CE phase is therefore similar to the case of a non-chirped laser waveform, except that the harmonic-like peaks become narrower due to the greater number of attosecond pulses interfering in time during the longer frequency-chirped pulse envelope (Fig. 2a).

\section{Controlling laser-driven plasmas}

In summary, our experiment is the first demonstration of attosecond control of collective electronic processes in laser-driven plasmas and with it the successful extension of attosecond optical techniques to plasma physics. Simply by spectrally resolving the coherent XUV radiation emitted by a solid illuminated by an intense fewcycle laser field of controlled waveform, we get an accurate temporal picture of the field-driven motion of charge through the thin layer of plasma formed at the surface. These experiments open the door to direct attosecond probing of the collective electronic response of a plasma to ultra-intense laser fields. As few-cycle laser pulses with orders of magnitude more peak-power will become accessible in the near future, our work is the first step towards controlling attosecond electronic processes in plasmas at relativistic light intensities, which is the key to developing ultrafast plasma-based particle accelerators and X-ray sources for applications.

\section{References}

[1] Esarey, E., Schroeder, C. B. \& Leemans, W. P. Physics of laser-driven plasma-based electron accelerators. Rev. Mod. Phys. 81, 1229-1285 (2009).

[2] Malka, V. et al. Principles and applications of compact laser-plasma accelerators. Nature Phys. 4, 447-453 (2008).

[3] Baltuska, A. et al. Attosecond control of electronic processes by intense light fields. Nature 421, 611-615 (2003).

[4] Uiberacker, M. et al. Attosecond real-time observation of electron tunnelling in atoms. Nature 446, 627-632 (2007).

[5] Eckle, P. et al. Attosecond Ionization and Tunneling Delay Time Measurements in Helium. Science 322, $1525-1529$ (2008). 
[6] Goulielmakis, E. et al. Real-time observation of valence electron motion, Nature 466. 739-743 (2010).

[7] Kling, M.F. et al. Control of Electron Localization in Molecular Dissociation. Science 312, 246-248 (2006).

[8] Sansone, G. et al. Electron localization following attosecond molecular photoionization. Nature 465, 763$766(2010)$.

[9] Cavalieri, A.L. et al. Attosecond spectroscopy in condensed matter. Nature 449, 1029-1032 (2007).

[10] Schultze, M. et al. Delay in Photoemission. Science 328, 1658-1662 (2010).

[11] Ivanov, M. \& Krausz, F. Attosecond physics. Rev. Mod. Phys. 81, 163-234 (2009).

[12] Heissler, P. et al. Toward single attosecond pulses using harmonic emission from solid-density plasmas. Appl. Phys. B 101, 511-521 (2010).

[13] Rolland, C. \& Corkum, P.B. Generation of 130-fsec midinfrared pulses. J. Opt. Soc. Am. B 3, 1625-1629 (1986).

[14] Kapteyn, H.C., Murnane, M.M., Szoke, A. \& Falcone, R.W. Prepulse energy suppression for high-energy ultrashort pulses using self-induced plasma shuttering. Opt. Lett. 16, 490-492 (1991).

[15] Doumy, G. et al. Complete characterization of a plasma mirror for the production of high-contrast ultraintense laser pulses. Phys. Rev. E 69, 026402 (2004).

[16] Dromey, B., Kar, S., Zepf, M. \& Foster, P. The plasma mirror - A subpicosecond optical switch for ultrahigh power lasers. Rev. Sci. Instrum. 75, 645-649 (2004).

[17] Thaury C. et al. Plasma mirrors for ultrahigh-intensity optics. Nature Phys. 3, 424-429 (2007).

[18] Brunel, F. Not-so-resonant, resonant absorption. Phys. Rev. Lett. 59, 52-55 (1987).

[19] Bonnaud, G., Gibbon, P., Kindel, J. \& Williams, E. Laser interaction with a sharp-edged overdense plasma. Laser Part. Beams 9, 339-354 (1991).

[20] Thaury, C. \& Quéré, F. High-order harmonic and attosecond pulse generation on plasma mirrors: basic mechanisms. J. Phys. B 43, 21300 (2010).

[21] Kruer, W.L. The physics of laser plasma interaction, Westview Press (2003).

[22] Hinkel-Lipsker, D.E., Fried, B.D. \& Morales, G.J. Analytic expressions for mode conversion in a plasma with a linear density profile. Physics of Fluids B 4, 559-575 (1992).

[23] Sheng, Z-M., Mima, K., Zhang, J. \& Sanuki, H. Emission of electromagnetic pulses from laser wakefields through linear mode conversion. Phys. Rev. Lett. 94, 095003 (2005).

[24] Quéré, F. et al. Coherent Wake Emission of High-Order Harmonics from Overdense Plasmas. Phys. Rev. Lett. 96, 125004 (2006). 
[25] Nomura, Y. et al. Attosecond phase locking of harmonics emitted from laser-produced plasmas, Nature Physics 5, 124-128 (2009).

[26] Borot, A. et al. High-harmonic generation from plasma mirrors at kilohertz repetition rate. Opt. Lett. 36, 1461-1463 (2011).

[27] Quéré, F. et al. Phase Properties of Laser High-Order Harmonics Generated on Plasma Mirrors. Phys. Rev. Lett 100, 095004 (2008).

[28] Thaury, C. et al. Coherent dynamics of plasma mirrors. Nat. Phys. 4, 631-634 (2008).

[29] Amidror, I The Theory of the Moiré Phenomenon Vol. I. Springer 2nd ed. (2009).

Acknowledgement: The authors would like to thank M. Ivanov for fruitful discussions, E. Lefebvre for providing the PIC code CALDER and R. Nuter for modifying this code to include the CE phase parameter. The 2D PIC calculations were performed using the computing resources of the "Grand Equipement National de Calcul Intensif" (GENCI), under project number 2011-056057, and those of the "Centre de Calcul Recherche et Technologie" (CCRT). Financial support was received from the Agence Nationale pour la Recherche through programs Chaire d'Excellence 2004 and ANR-09-JC-JC-0063 (UBICUIL). A.B. acknowledges financial support from the RTRA - Triangle de la Physique and F.Q. from the European Research Council (ERC grant agreement $\left.\mathrm{n}^{\circ} 240013\right)$.

Authors contributions: The experimental setup was designed by A.B. and R.L-M., the experiments were carried out by A.B. and A.M. The theoretical work was done by A.M. and F.Q. 


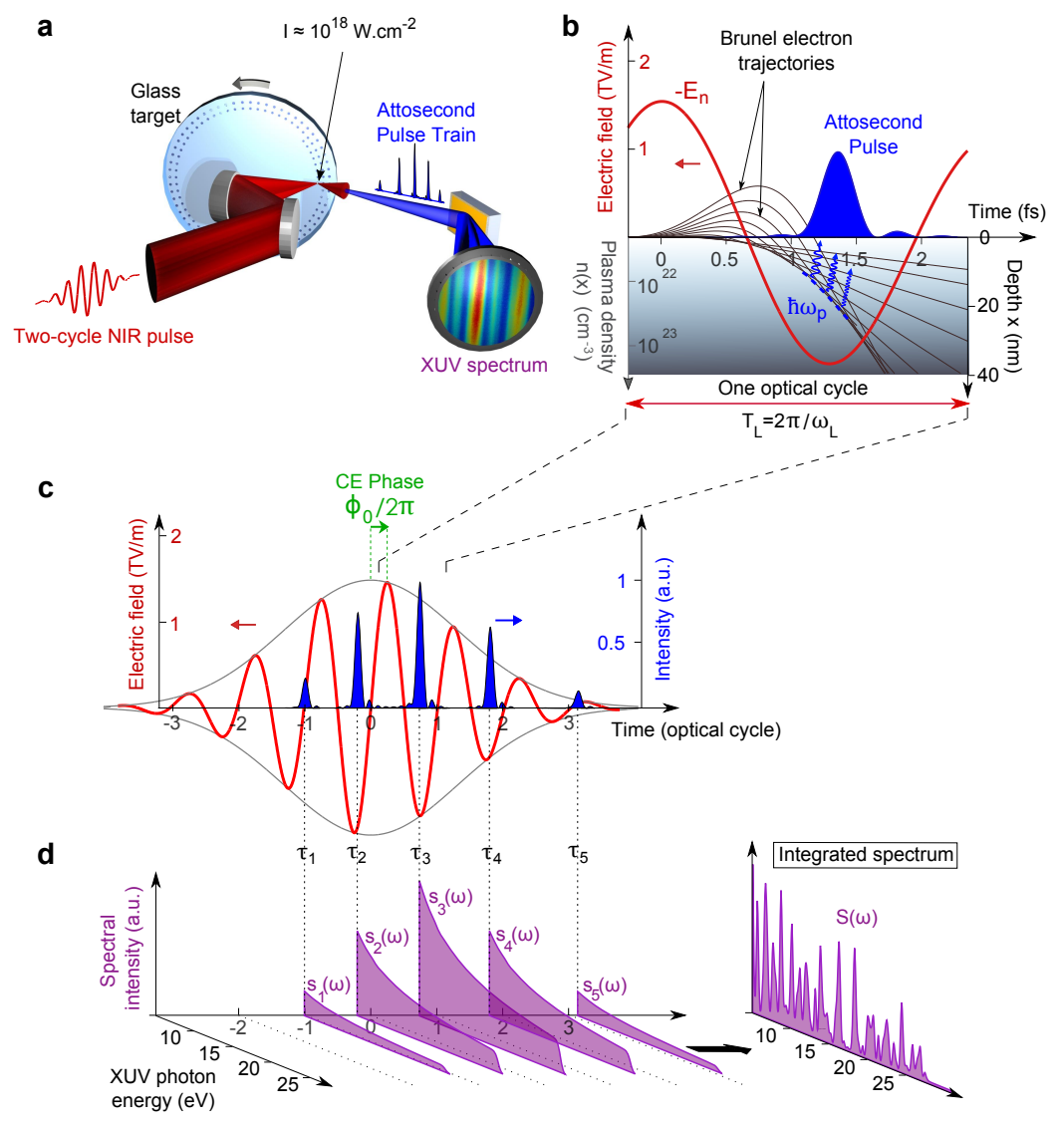

Figure 1: Schematic and principle of the experiment. (a) experimental set-up. Controlled few-cycle NIR light waveforms are focused at $1 \mathrm{kHz}$ repetition rate onto a rotating glass surface [26] at a peak intensity around $10^{18} \mathrm{~W} / \mathrm{cm}^{2}$. The coherent XUV radiation emitted by the plasma created at the target surface is spectrally resolved. (b) Sketch of the charge dynamics at the plasma surface within a single NIR light cycle. The intense NIR electric-field (red) first pulls electrons out of the plasma and then pushes them back into the dense plasma layer, where their different trajectories (black lines) cross to form a few-100-as charge density wave that triggers background plasma oscillations in its wake. These oscillations radiate light in the form of attosecond XUV bursts (blue) with spectral components $\omega_{p}$ dependent on the plasma density (bottom left axis). (c) Sketch of the attosecond pulse train (APT, blue) generated by a two-cycle NIR waveform (red) with arbitrary CE phase $\phi_{0}$. The unevenly spaced emission times $\tau_{j}$ show the influence of the light waveform on the time structure of the APT. (d) Build-up of the time-integrated spectrum. The purple areas $s_{j}(\omega)$ show the spectra of the individual attosecond bursts for each laser cycle. The total measured spectrum $S(\omega)$ results from the spectral interference of all the XUV bursts produced during the laser pulse. 


\section{Experiment}
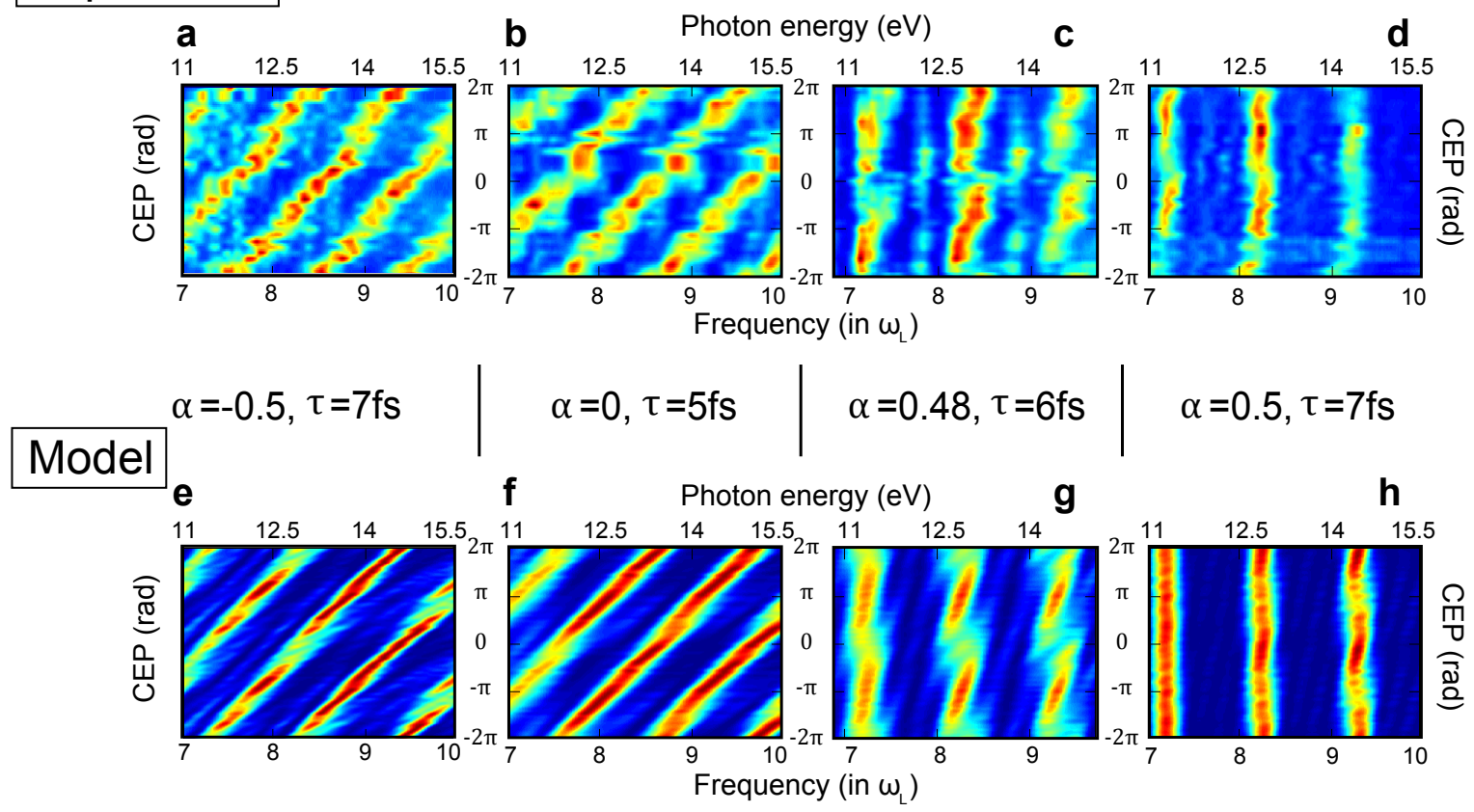

Figure 2: Waveform-dependent plasma emission spectra. Experimental (top row) and modeled (bottom row) CWE spectra obtained for different few-cycle NIR light waveforms (CE phase and linear frequency chirp) with 0.8 micron central wavelength. The experimental setup allows us to record XUV photon energies ranging from 11 and $15.5 \mathrm{eV}$, corresponding to harmonics 7 to 10 of the central frequency $\omega_{L}$ of the NIR laser. The relative CE phase scale of the experimental scans is calibrated against the absolute CE phase values used in the model assuming an exponentially decaying plasma density gradient of $\lambda_{L} / 100$ at the target surface and a peak intensity for a non-chirped pulse of $4 \times 10^{17} \mathrm{~W} / \mathrm{cm}^{2}$. 

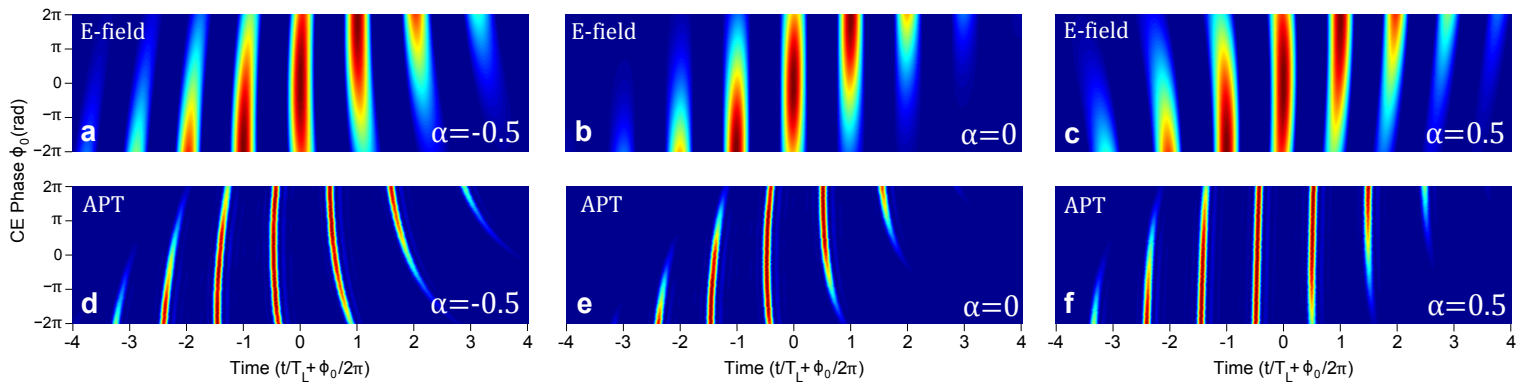

Figure 3: Attosecond metrology of the plasma emission. Attosecond pulse emission time for controlled two-cycle (5-fs Fourier limit) NIR light waveforms with different frequency chirps. The waveforms corresponding to different frequency chirps are displayed in $(\mathbf{a}),(\mathbf{b})$ and $(\mathbf{c})$, where for clarity only the positive values of the NIR light wave oscillations are shown. Both the NIR electric-field and corresponding temporal intensity profiles of the generated attosecond pulse trains displayed in ((d) to (f)) are plotted as a function of $\bar{t}=t / T_{L}+\phi_{0} / 2 \pi$, where $\phi_{0}$ is the CE phase of the light field with respect to the few-cycle pulse envelope. This representation shows how the whole attosecond puse train shifts in time as the CE phase is swept. A negative chirp (a and d) increases the naturally uneven spacing between the attosecond pulses (subcycle emission time) in the APT observed for zero chirp ( $\mathbf{b}$ and $\mathbf{e}$ ). In contrast, a positive chirp (c and $\mathbf{f}$ ) leads to an evenly spaced APT with weak CE phase dependence. 

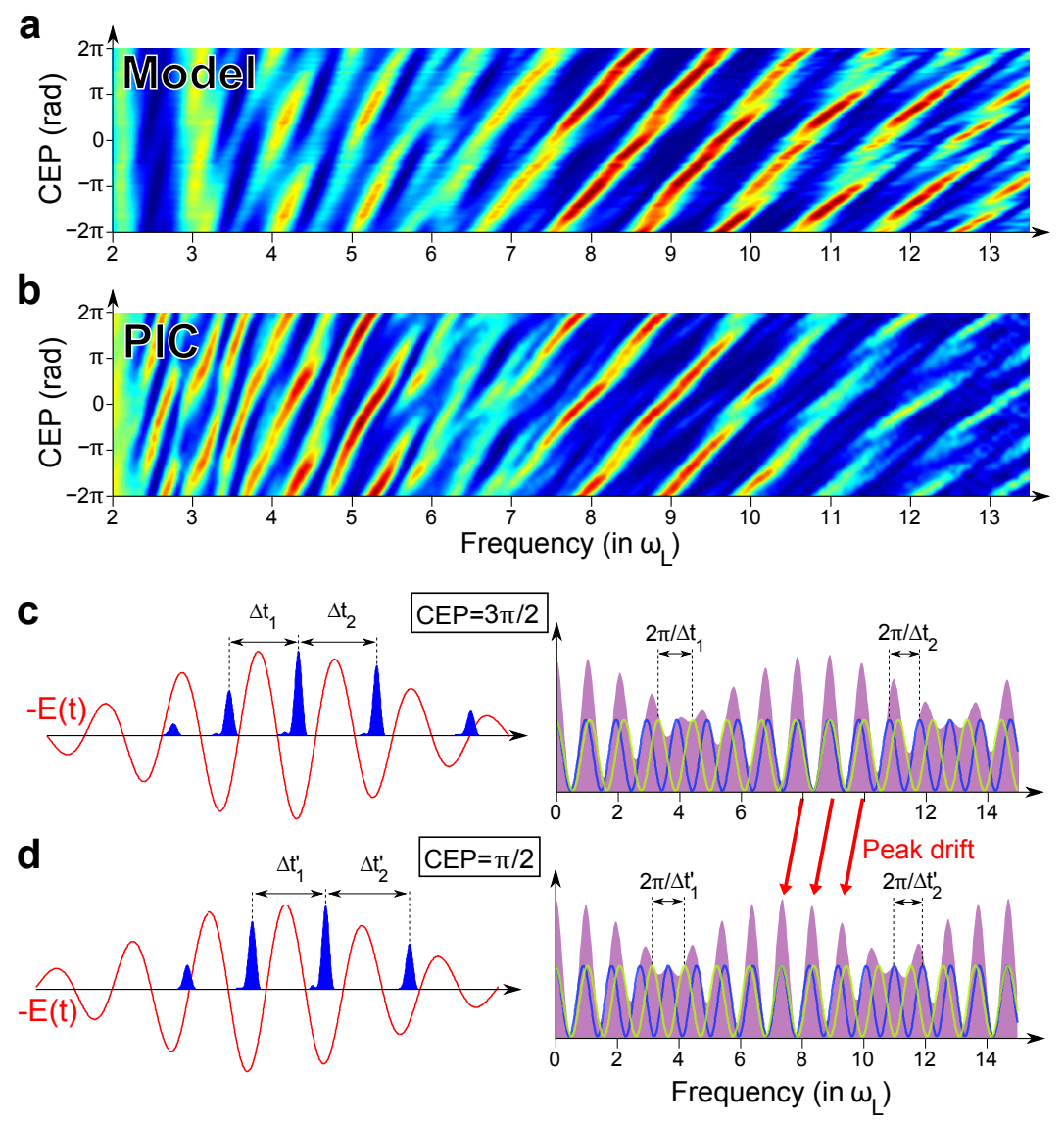

Figure 4: Moiré patterns in plasma emission spectra. (a) 1D model and (b) 2D PIC simulation of CWE spectra as a function of the CE phase of a two-cycle (5-fs Fourier limit) NIR pulse in the same conditions as described in Fig. 2B. The spectrum consists of a region with well-contrasted harmonic-like peaks surrounded by intervals with lower contrast but still a periodic CE phase dependence. This corresponds to the moiré pattern produced by the spectral interference between the unevenly spaced attosecond pulses displayed in (c) and (d) left, for two different CE phase values. The moiré pattern resulting from the interference between the three most intense pulses in the APT is displayed in (c) and (d), right. The beating of the sinusoidal waves with slightly different frequencies resulting from the different timing between adjacent attosecond pulses leads to a blurring of the overall signal except when waves happen to be in phase. Moreover, as the CE phase is decreased from $(\mathbf{c})$ to $(\mathbf{d})$, the spacing between the attosecond pulses increases, thereby shifting the constructive interference pattern to lower harmonic frequencies and giving rise to the linear drift observed in the experiment. 